\title{
Controlled manipulation of multiple cells using catalytic microbots $\dagger$
}

\author{
Samuel Sanchez, ${ }^{* a}$ Alexander A. Solovev, ${ }^{a}$ Sabine Schulze ${ }^{a b}$ and Oliver G. Schmidt ${ }^{a}$ \\ Received 29th September 2010, Accepted 26th October 2010 \\ DOI: $10.1039 / \mathrm{c0cc04126b}$
}

Self-propelled microjet engines (microbots) can transport multiple cells into specific locations in a fluid. The motion is externally controlled by a magnetic field which allows to selectively load, transport and deliver the cells.

The development of useful micro- or nanomachines which could one day be manipulated inside the human body remains a challenging dream in nanotechnology and biomedicine. ${ }^{1}$ Over the last five years, there has been substantial interest in the use of chemistry to propel tiny engines in a similar fashion that nature uses biochemistry to power biological motors. ${ }^{2,3}$ Consequently, researchers have fabricated self-propelled nanomachines capable of performing useful tasks such as the transport of synthetic cargoes. ${ }^{4,5}$ Artificial nanomachines that are able to swim along with biological matter such as cells have yet to be achieved.

There are three main challenges that researchers try to conquer when engineering artificial nanomachines: (i) efficient self-propulsion, demonstrated by the catalytic breakdown of $\mathrm{H}_{2} \mathrm{O}_{2}$ by $\mathrm{Pt},{ }^{3,6} \mathrm{Ni}^{7,8}$ catalysts and catalase enzyme ${ }^{9}$ contained in nanomotors; (ii) motion control, achieved by the incorporation of $\mathrm{Ni}$ or $\mathrm{Fe}$ segments and subsequently using external magnetic fields to orient the nanomotors ${ }^{4,5,10,11}$ and; (iii) the development of useful task such as the transport of cargo like microparticles and nanoplates in a fluid., ${ }^{4,5,12}$ To date, bimetallic nanomotors ${ }^{4}$ and tubular microjet engines ${ }^{5,12}$ have achieved these three requisites. However, the transport of a single spherical microparticle has been the last result accomplished by the nanomotors. ${ }^{4}$ On the other hand, microjet engines (dubbed microbots) based on rolled-up nanotechnology have demonstrated the ability to transport multiple microobjects, not only colloidal microparticles but also metallic nanoplates. ${ }^{5}$ Nonetheless, the transport of biological material such as cells by artificial nanomachines has not been achieved so far.

Here we report on the pick-up, transport and release, of multiple neuronal CAD cells (cathecolaminergic cell line from the central nervous system) in a fluid by using catalytic microbots. Although "large" cells are loaded at the front end of the microbots, their motion is not totally halted. The microengines are self-propelled by the release of oxygen bubbles generated in the cavity of the microtubes ( $\mu$-tubes)

\footnotetext{
${ }^{a}$ Institute for Integrative Nanosciences, IFW Dresden,

Helmholtzstr 20, D-01069 Dresden, 01069, Germany.

E-mail: s.sanchez@ifw-dresden.de; Fax: +493514659 782;

Tel: +493514659845

${ }^{b}$ Institute of Physiological Chemistry, Medical Faculty Carl Gustav Carus Dresden, University of Technology, Fiedlerstraße 42, D-01307 Dresden, Germany

$\dagger$ Electronic supplementary information (ESI) available: Videos illustrating the microbot loading multiple CAD cells are available.
}

from the catalytic decomposition of peroxide used as fuel. Their motion is coordinated by an external magnetic field, which-once is turned rapidly-enables the release of the loaded cell at a desired target. To the best of our knowledge, this is the first report on the transportation of cells using any kind of artificial micro-nanomachine (nanomotor or microengine). The controlled transport of cells is of significant importance since it is clearly the next step towards the use of artificial nanomachines in future biomedical applications.

$\mathrm{Ti} / \mathrm{Fe} / \mathrm{Pt} \mu$-tubes were fabricated following the wellestablished rolled-up technique developed by our group. ${ }^{13,14}$

First, silicon substrates were patterned with a photoresist layer of square structures of $50 \mu \mathrm{m} \times 50 \mu \mathrm{m}$ in size. Photoresist AR-P 3510 was spin-coated on Si wafers at $3500 \mathrm{rpm}$ for $35 \mathrm{~s}$, followed by a baking step and exposure to UV light with a Karl Suss MA-56 mask aligner. The subsequent structure was developed in an AR300-35: $\mathrm{H}_{2} \mathrm{O}$ solution (1: 1).

The nanomembranes were then deposited by electron-beam (Ti and $\mathrm{Fe}$ ) and magnetron sputtering $(\mathrm{Pt})$, respectively. Thereafter, the structures were released from the substrate by removing the sacrificial layer (photoresist) with acetone, which causes the deposited nanomembranes to roll-up into $\mu$-tubes of $50 \mu \mathrm{m}$ in length. A supercritical point drier is needed to avoid collapsing of the tube during drying due to the high surface tension of the etchant.

On-demand and mass production of microbots is easily achieved with the rolled-up technique. In particular, the diameter of the $\mu$-tubes can be tailored by changing the thicknesses and the built-in strain of the deposited layers. ${ }^{14,15}$ This versatility is of great interest concerning the transportation of cells with different diameters. For instance, the typical cell size varies from about $1 \mu \mathrm{m}$ for some bacteria, $4 \mu \mathrm{m}$ for yeast cells, $8 \mu \mathrm{m}$ for red blood cells, $10 \mu \mathrm{m}$ for animal cells or $100 \mu \mathrm{m}$ for plant cells. Therefore, the fabrication of $\mu$-tubes with variable diameters is of significant importance in order to manipulate cells on-demand in an accurate manner.

The murine CAD cells were grown in DMEM/F-12 medium (Dulbecco's Modified Eagle Medium) supplemented with 10\% FCS (Fetal Calf serum) and 1\% penicillin/streptomycin. The cells were incubated in a humidified atmosphere with $5 \% \mathrm{CO}_{2}$ and were passaged every 3-4 days by trypsination and centrifugation at $4400 \mathrm{rpm}$ for $5 \mathrm{~min}$. After determination of cell numbers with a hemocytometer, CAD cells were plated at $10^{5}$ cells per plate. A small amount of CAD cells $(10 \mu \mathrm{l}$ of $\sim 10^{5}$ cells per $\mathrm{ml}$ ) was suspended in $1.2 \mathrm{ml}$ of the working solution containing the microbots and the chemical fuel to actuate them.

The microbots are formed by a hollow tubular structure containing a thin Pt layer in their inside. When the $\mu$-tubes are immersed into a hydrogen peroxide solution, microbubbles are generated in one or more nucleation points of the Pt layer due 


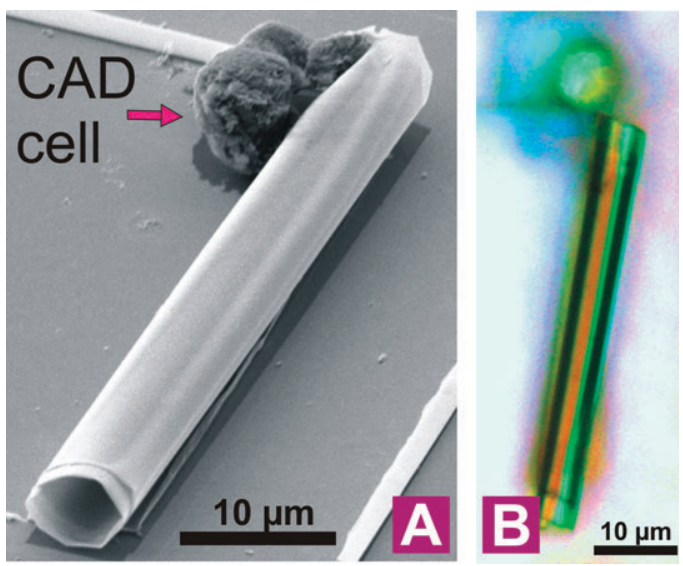

Fig. 1 SEM (A) and optical (B) images of $\mathrm{Ti} / \mathrm{Fe} / \mathrm{Pt}$ rolled-up $\mu$-tubes with CAD cells.

to the catalytic decomposition of $\mathrm{H}_{2} \mathrm{O}_{2}$ into water and molecular di-oxygen. These oxygen bubbles grow and expand into the microtube, and their recoil from one of the openings thrusts the microtube. The migration of the bubbles inside the tube induces a pumping of the fluid, as described previously elsewhere. ${ }^{5}$

As a proof-of-concept, we manipulated murine CAD cells with diameters ranging from 10 to $15 \mu \mathrm{m}$. One would expect that a microbot with diameter larger than $15 \mu \mathrm{m}$ will suck the cell through the tube body due to the fluid pumping, whereas a microbot with a very small size will not be suitable for the manipulation of a very large cell. Therefore, we optimized the fabrication parameters to produce microbots with diameters from 6 to $10 \mu \mathrm{m}$. This was achieved by depositing $10 \mathrm{~nm}$ of Ti and Fe (electron beam) and $1 \mathrm{~nm}$ of $\mathrm{Pt}$ (sputtering). The dimensionality of the fabricated microbots compared to CAD cells is clearly revealed by SEM (Fig. 1A) and optical imaging (Fig. 1B).

The transport (i.e. towing) of microparticles using bimetallic nanomotors was only achieved with single cargoes ranging from 0.8 to $4.3 \mu \mathrm{m} .{ }^{4}$ Furthermore, these nanomotors always required either pre-loading functionalization or were limited to magnetic particles. Additionally, low towing power has rendered the loading of cells a challenging task still unattained. We recently demonstrated the ability to load larger amounts of polysterene microparticles mediated directly by our microbots. ${ }^{5}$

Here we extend the ability of the microbots to be remotely controlled by a magnetic field. We directed the microbots towards the suspended cells, which undergo apoptosis in these conditions (Fig. 2A), pick-up the cells, transport them (Fig. 2B) until a desired location is reached.

By a rapid turn of the magnet, the cells are quickly and easily released from the tube (Fig. 2C).

In the optical sequences shown in Fig. 2, the microbot swims along pushing the cells over a period of about $9 \mathrm{~s}$ and after releasing them, continues to travel in the solution to re-load other cells. A long working time of catalytic multi-task microbots allows their operation for several hours. Video 1 corresponding to Fig. 2 is available in ESI. $\dagger$

Fig. 3 illustrates the microbot speed and angle of rotation during the loading, transport and delivery of the CAD cells. The microbots are self-propelled in $4 \%$ peroxide containing $0.005 \mathrm{w} / \mathrm{w} \%$ of benzalkonium chloride and $25 \%$ of cells medium. The initial speed, i.e. before loading cells, is $130 \mu \mathrm{m} \mathrm{s}^{-1}$ and it

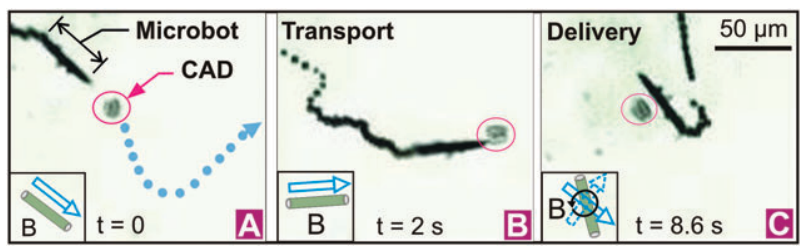

Fig. 2 Controlled manipulation of CAD cells by using catalytic microbots. The motion of the microbot is aligned by an external magnetic field (schematic insets) provided by a small magnet which is placed underneath the sample containing the cells and microbots. (A) Microbot directed towards the CAD cell, its transport (B) and delivery in a desired location by a quick rotation of the magnet (C).

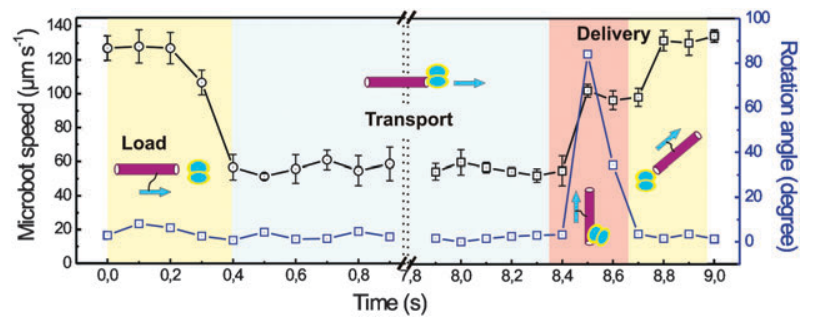

Fig. 3 Speed of the microbot (left $y$ axis, black circles) and angle of rotation (right $y$ axis, blue line) during the process of loading, transport and delivery of CAD cells. Background colours: yellow, microbot free of cells; grey, transporting two cells; orange, delivering cells into defined location.

reduces dramatically to $60 \mu \mathrm{m} \mathrm{s}^{-1}$ shortly after the loading of CAD cells (Fig. 3, left $y$ axis). The main reason of the deceleration on the microbot speed is due to the increase on the drag force exerted by the "large" loaded cells. During the next $8 \mathrm{~s}$. the speed does not change significantly until the magnetic field is abruptly turned $90^{\circ}$ within $0.2 \mathrm{~s}$ (Fig. 3, right $y$ axis) leading to the delivery of the cells (detachment) and consequently an increase of the speed up to $70 \mu \mathrm{m} \mathrm{s}^{-1}$ is observed. It is important to note that the cells remain loosely attached to the wall of the microbot. Shortly after the first turn, thus, another 'shake' of $40^{\circ}$ is necessary to achieve the total delivery of the cell (see also video $1, \mathrm{ESI} \dagger$ ).

Sophisticated micromachines are required for the performance of multiple tasks. For instance, the consecutive loading of several cells is beneficial for complex applications such as cell sorting. Therefore, high power output and easy loading mechanisms are demanded for this purpose. The loading of multiple CAD cells during a period from 10 to $20 \mathrm{~s}$ is shown in Videos 2, 3 and 4 in ESI. $\dagger$ The microbots can overcome the drag force generated by the loaded cells. Although their speed is reduced, the microbots can travel at speeds of roughly $40 \mu \mathrm{m} \mathrm{s}^{-1}$ while transporting large biological material (analysis from video 3 and video 4, ESI $\dagger$ ). Our experiments clearly indicate that our microbots could be suitable in the near future for the development of bioanalytical and biomedical applications using different kind of cells.

In summary, we have demonstrated that catalytic microbots can transport animal cells in a controllable manner and release them to desired targets. The presented work paves the way for future biomedial applications by using artificial nano-micromachines. For instance, the microbots can be used for cell sorting or to move one single cell into an analytical 
device (e.g., lab-on-a-chip) to analyse the changes in biochemistry, physiology or metabolism of the single cell. One could foresee that in not-too-distant future such powerful microbots could transport drugs to cure unhealthy cells. Other visionary tasks could be the separation of cancer cells, the translocation of stem cells on-demand to build up new tissues or to replace disease cells or tissues.

The authors acknowledge the Volkswagen Foundation for the grant (I/84 072). The authors' thanks go to S. Harazim and D. Thurmer for fruitful discussion, and to Prof. Y.F. Mei for comments.

\section{Notes and references}

1 T. E. Mallouk and A. Sen, Sci. Am., 2009, 300, 72; G. A. Ozin, I. Manners, S. Fournier-Bidoz and A. Arsenault, Adv. Mater., $2005,17,3011$.

2 T. Mirkovic, N. S. Zacharia, G. D. Scholes and G. A. Ozin, Small, 2010, 6(2), 159.

3 M. Pumera, Nanoscale, 2010, 2, 1643; W. Paxton, S. Sundararajan, T. Mallouk and A. Sen, Angew. Chem., Int. Ed., 2006, 45, 5420; A. Sen, M. Ibele, Y. Hong and D. Velegol, Faraday Discuss., 2009, 143, 15; S. J. Ebbens and J. R. Howse, Soft Matter, 2010, 6, 726; S. Sanchez and M. Pumera, Chem.-Asian J., 2009, 4, 1402.
4 S. Sundararajan, P. E. Lammert, A. W. Zudans, V. H. Crespi and A. Sen, Nano Lett., 2008, 8, 1271; J. Burdick, R. Laocharoensuk, P. M. Wheat, J. D. Posner and J. Wang, J. Am. Chem. Soc., 2008, 130, 8164 .

5 A. A. Solovev, S. Sanchez, M. Pumera, Y. F. Mei and O. G. Schmidt, Adv. Funct. Mater., 2010, 20, 2430.

6 W. F. Paxton, K. C. Kistler, C. C. Olmeda, A. Sen, S. K. St. Angelo, Y. Cao, T. E. Mallouk, P. E. Lammert and V. H. Crespi, J. Am. Chem. Soc., 2004, 126, 13424.

7 S. Fournier-Bidoz, A. C. Arsenault, I. Manners and G. A. Ozin, Chem. Commun., 2005, 441.

8 N. S. Zacharia, Z. S. Sadeq and G. A. Ozin, Chem. Commun., 2009, 5856.

9 S. Sanchez, A. A. Solovev, Y. F. Mei and O. G. Schmidt, J. Am. Chem. Soc., 2010, 132, 13144.

10 T. R. Kline, W. F. Paxton, T. E. Mallouk and A. Sen, Angew. Chem., Int. Ed., 2005, 44, 744.

11 A. A. Solovev, Y. F. Mei, E. Bermudez Urena, G. Huang and O. G. Schmidt, Small, 2009, 5, 1688.

12 K. M. Manesh, R. Yuan, M. Clark, D. Kagan, S. Balasubramanian and J. Wang, ACS Nano, 2010, 4, 1799.

13 O. G. Schmidt and K. Eberl, Nature, 2001, 410, 168.

14 Y. F. Mei, G. S. Huang, A. A. Solovev, E. Bermudez Urena, I. Monch, F. Ding, T. Reindl, R. K. Y. Fu, P. K. Chu and O. G. Schmidt, Adv. Mater., 2008, 20, 4085.

15 C. Deneke, C. Müller, N. Y. Jin-Phillipp and O. G. Schmidt, Semicond. Sci. Technol., 2002, 17, 1278. 